\title{
A SEARCH FOR MAGNESIUM IN EUROPA'S ATMOSPHERE
}

\author{
S. M. HÖRST ${ }^{1}$ AND M. E. BROWN ${ }^{2}$ \\ ${ }^{1}$ Cooperative Institute for Research in Environmental Sciences, University of Colorado-Boulder, Boulder, CO, USA; sarah.horst@ colorado.edu \\ ${ }^{2}$ Division of Geological and Planetary Sciences, California Institute of Technology, Pasadena, CA, USA \\ Received 2013 January 14; accepted 2013 January 26; published 2013 February 7
}

\begin{abstract}
Europa's tenuous atmosphere results from sputtering of the surface. The trace element composition of its atmosphere is therefore related to the composition of Europa's surface. Magnesium salts are often invoked to explain Galileo Near Infrared Mapping Spectrometer spectra of Europa's surface, thus magnesium may be present in Europa's atmosphere. We have searched for magnesium emission in the Hubble Space Telescope Faint Object Spectrograph archival spectra of Europa's atmosphere. Magnesium was not detected and we calculate an upper limit on the magnesium column abundance. This upper limit indicates that either Europa's surface is depleted in magnesium relative to sodium and potassium, or magnesium is not sputtered as efficiently resulting in a relative depletion in its atmosphere.
\end{abstract}

Key words: planets and satellites: atmospheres - planets and satellites: composition

\section{INTRODUCTION}

Jupiter's moon Europa likely possesses a global subsurface salty ocean (Anderson et al. 1998; Carr et al. 1998; Kivelson et al. 2000). Although we cannot currently directly measure the ocean composition, if the ocean is in communication with the surface then evaporites may be present on the surface where their composition is accessible to remote sensing. Knowledge of the surface composition therefore plays an important role in ascertaining whether, and to what extent, the subsurface ocean communicates with the surface, and if the ocean is in communication with the surface, then the surface composition should also provide information about composition of the internal ocean.

The Near Infrared Mapping Spectrometer (NIMS), carried by the Galileo spacecraft (Carlson et al. 1992), obtained spectra of Europa's surface from 0.7 to $5.2 \mu \mathrm{m}$ and found evidence for the spectral signatures of abundant non-water ice material. These spectral signatures were initially interpreted as indicative of the presence of hydrated salts, such as hexahydrite $\left(\mathrm{MgSO}_{4} \cdot 6 \mathrm{H}_{2} \mathrm{O}\right)$ and epsomite $\left(\mathrm{MgSO}_{4} \cdot 7 \mathrm{H}_{2} \mathrm{O}\right)$, assumed to originate from a subsurface ocean (McCord et al. 1998, 2001), but it was later shown that hydrated sulfuric acid $\left(\mathrm{H}_{2} \mathrm{SO}_{4} \cdot \mathrm{n}-\mathrm{H}_{2} \mathrm{O}\right)$, an expected product of Europa's irradiation, could also account for the spectral signatures (Carlson et al. 1999, 2002; Strazzulla et al. 2007). The presence of other sulfur species $\left(\mathrm{SO}_{2}\right.$ and sulfur allotropes) detected on the surface, primarily on the heavily bombarded trailing hemisphere (Lane et al. 1981; Carlson et al. 2009; Paranicas et al. 2001, 2002) indicates that chemistry driven by sulfur ions from Io affects the chemical composition of the surface. However, unambiguous interpretation of the NIMS spectra is not possible due to their relatively low resolution, which precludes the presence of distinct spectral features that would uniquely identify surface constituents (Carlson et al. 2009). The relative contribution, if any, of evaporites from the subsurface ocean and the composition of those evaporites is still unknown.

The atmosphere of Europa results primarily from sputtering of the surface by energetic particles from the Jovian magnetosphere. The composition of the atmosphere strongly reflects the composition of the surface; examination of Europa's atmosphere can be used to investigate its surface. Since the surface is dominated by water ice, the principal constituents of Europa's atmosphere are water and its products (see, e.g., McGrath et al. 2009). The atmosphere also contains traces of other elements; both sodium and potassium have been detected in Europa's atmosphere (Brown \& Hill 1996; Brown 2001). While the detection of sodium and potassium could indicate the presence of salts on Europa's surface, it is also possible that they are simply contaminants carried from Io (Brown \& Hill 1996). The sodiumto-potassium ratio at Europa is higher than that at Io, contrary to the expectation from simple contamination (Brown 2001). However, as Carlson et al. (2009) point out that the details of implantation and sputtering are sufficiently uncertain that the ratio difference is not a definitive test.

In more recent spectral models of NIMS data, magnesium sulfates dominate much of the non-water ice component of the surface (Dalton et al. 2005, 2012; Dalton 2007; Dalton \& Pitman 2012). Such an enrichment of magnesium on the surface should result in a corresponding enrichment in the sputtered atmosphere of Europa. Here we use archival spectra from the Hubble Space Telescope Faint Object Spectrograph (HST-FOS) to search for the signature of the strong $2852 \AA$ resonant scattering emission line of magnesium in Europa's atmosphere. Comparison with known abundances of sodium and potassium in the atmosphere allows us to place constraints on the presence of magnesium on the surface of Europa.

\section{OBSERVATIONS AND DATA REDUCTION}

The HST observed Europa on 1994 July 4 and 1994 July 29 using the FOS. The data include two $35 \mathrm{~s}$ spectra centered on Europa, five $924 \mathrm{~s}$ spectra offset 8.8 Europa radii $\left(R_{E}\right)$ south of Europa, and three $1100 \mathrm{~s}$ spectra offset $14.4 R_{E}$ south of Europa. The spectra were obtained using the blue detector and the A1 aperture, which is $3^{\prime \prime} 66 \times 1^{\prime \prime} .29\left(1.11 \times 10^{-10} \mathrm{SR}\right)$. FOS was operated in the image mode using the $\mathrm{G} 270 \mathrm{H}$ grating. The spectra cover 2200 to $3200 \AA$, which includes the magnesium emission line at $2852 \AA$. Table 1 contains details of the observations.

Due to problems with the data headers, the archival data are not correctly processed by the Space Telescope Science Institute's automated calibration pipeline POA CALFOS. We instead process the data manually using the provided post operational archive calibration files. We convert the raw count 


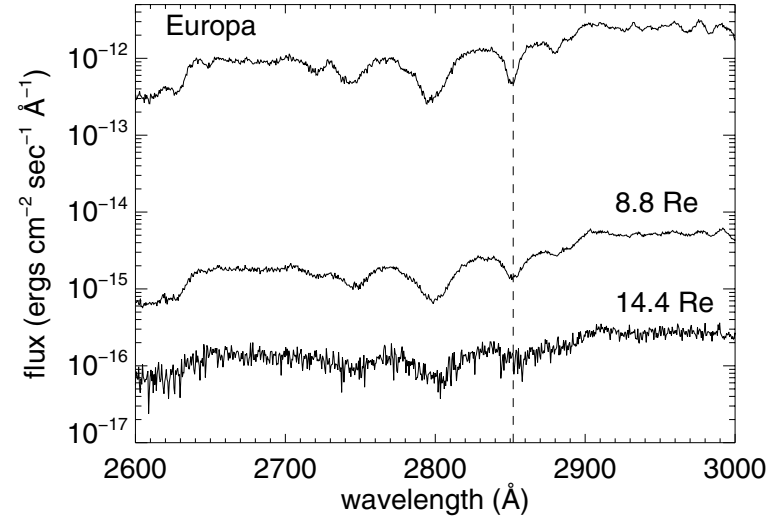

Figure 1. HST-FOS spectra of Europa and its atmosphere. The top spectrum was obtained centered on Europa (top), while the middle and bottom spectra were obtained offset by $8.8 R_{E}$ and $14.4 R_{E}$ respectively. The dashed line indicates the wavelength of the $\mathrm{Mg}$ resonant scattering line at $2852 \AA$.

Table 1

$H S T$-FOS Observations of Europa

\begin{tabular}{lccccc}
\hline \hline Date & $\begin{array}{c}\text { Time } \\
(\text { UTC) }\end{array}$ & $\begin{array}{c}\text { Offset from } \\
\text { Europa }(\operatorname{arcsec})\end{array}$ & $\begin{array}{c}\text { Exp } \\
(\mathrm{s})\end{array}$ & $\begin{array}{c}\dot{r} \\
\left(\mathrm{~km} \mathrm{~s}^{-1}\right)\end{array}$ & $\begin{array}{c}\text { Phase } \\
\left({ }^{\circ}\right)\end{array}$ \\
\hline 1994 Jul 4 & $3: 58$ & 0 & 35 & 12.62 & 263.12 \\
1994 Jul 4 & $4: 05$ & 3.85 & 924 & 12.65 & 264.17 \\
1994 Jul 4 & $5: 30$ & 3.85 & 924 & 13.01 & 270.16 \\
1994 Jul 4 & $5: 50$ & 3.85 & 924 & 13.08 & 271.56 \\
1994 Jul 4 & $7: 06$ & 3.85 & 924 & 13.25 & 276.91 \\
1994 Jul 4 & $7: 27$ & 3.85 & 924 & 13.27 & 278.39 \\
1994 Jul 29 & $4: 17$ & 5.86 & 1100 & 13.27 & 278.09 \\
1994 Jul 29 & $5: 36$ & 5.86 & 1100 & 13.23 & 283.65 \\
1994 Jul 29 & $6: 00$ & 5.86 & 1100 & 13.32 & 285.34
\end{tabular}

Note. All observations used the G270H grating, image mode, blue detector, and A1 aperture.

rate of the spectrograph, in counts $\mathrm{s}^{-1}$ diode $^{-1}$, to the standard units of ergs $\mathrm{cm}^{-2} \mathrm{~s}^{-1} \AA^{-1}$ by first correcting for diode-to-diode sensitivity variations by multiplying by the flatfield response file and then multiplying by the inverse sensitivity curves. No attempt is made at background subtraction, as we fit and remove scattered light and background in our subsequent analysis. We confirmed our manual reduction routines by performing the same procedures on data that was processed with POA CALFOS.

Figure 1 shows the spectrum centered on Europa and the spectra at the two offset positions. The spectra at both offset positions, while orders of magnitude fainter than that of Europa, are dominated by scattered light from Europa. Visual inspection of the raw FOS spectra does not reveal any emission features from Europa's atmosphere. As can be seen from Figure 1, the scattered light fills the aperture more fully than the 0.87 arcsecond diameter Europa, and spectral lines are thus broader. Depending on the spatial structure of the scattered light, there is also a chance that the scattered light would appear shifted in wavelength compared to the Europa spectrum. Finally, the possibility exists of stray light scattered in the spectrograph. To account for these factors, we fit each scattered light spectrum with a broadened, shifted, scaled spectrum of Europa with a constant background added. We perform a $\chi^{2}$ minimization to find the best fit which minimizes the residuals between 2600 and $3000 \AA$ while excluding the region within $20 \AA$ of the expected $2852 \AA$ resonant scattering line. Figure 2 shows the spectra at the offset locations before and after subtraction of scattered light.

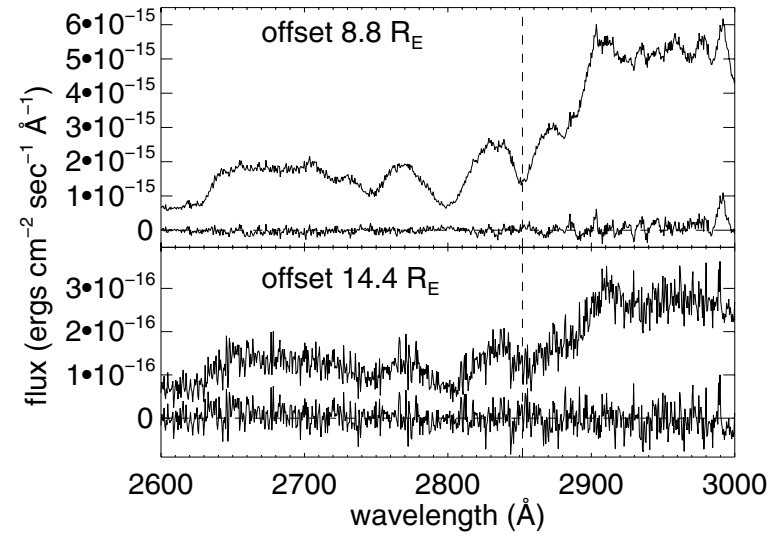

Figure 2. Spectra of Europa's extended atmosphere before and after subtraction of scattered light. The dashed line indicates the wavelength of the $\mathrm{Mg}$ resonant scattering line at $2852 \AA$.

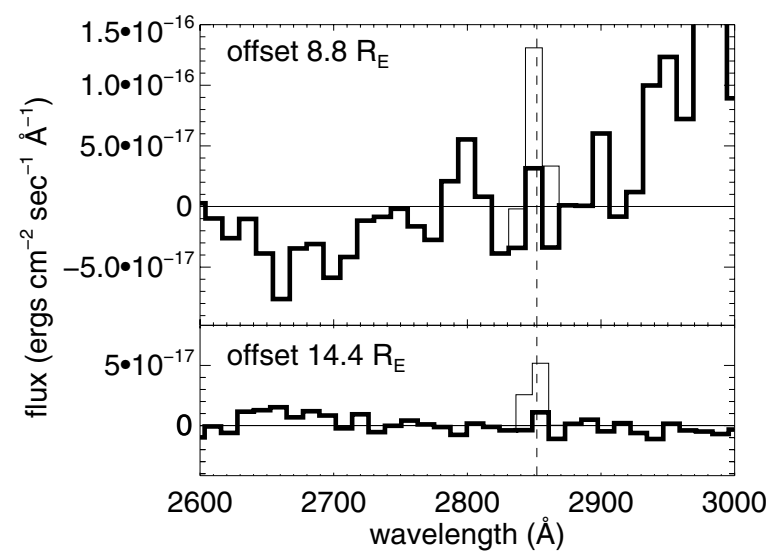

Figure 3. Background subtracted spectra binned to the resolution of an emission line filling the aperture (thick lines). The dashed line shows the wavelength of the $2852 \AA$ resonant scattering line of magnesium. The thin lines show the minimum detectable flux and are the basis of our upper limits.

The subtracted spectra are generally excellent in most regions, but do show some uncorrected systematic variations.

\section{DATA AND RESULTS}

Atmospheric emission from Europa will fill the FOS aperture, causing the monochromatic emission line to spread over the full 12 diode width of the A1 aperture, which corresponds to a line width of $\sim 24 \AA$. To better see such a broad emission line, we bin the data to $12 \AA$ per pixel. A line filling the aperture will thus have a 2 pixel width in the binned spectrum. Figure 3 shows the binned spectra at the two offset positions. No emission at the $2852 \AA$ location of the resonant scattering line of magnesium can be seen. Because of the uncorrected systematic errors in the subtraction, we cannot compute a statistically rigorous upper limit to the presence of an emission line, but we instead simply add artificial emission at $2852 \AA$ until it reaches a point where we would confidently declare a detection. This modeled lines that would be detectable are shown in Figure 3. Our upper limits to monochromatic emission at $2852 \AA$ in the offset spectra are $2.5 \times 10^{-15} \mathrm{erg} \mathrm{cm}^{-1} \mathrm{~s}^{-1}$ at $8.8 R_{E}$ and $1.0 \times 10^{-15} \mathrm{erg} \mathrm{cm}^{-1} \mathrm{~s}^{-1}$ at $14.4 R_{E}$, or an emission strength of $40 R$ at $8.8 R_{E}$ and $16 R$ at $14.4 R_{E}\left(1\right.$ Rayleigh $(R)=10^{6} / 4 \pi \times$ photons $\left.\mathrm{cm}^{-2} \mathrm{~s}^{-1} \mathrm{SR}^{-1}\right)$.

We converted the intensity upper limits to upper limits on the magnesium column density using a photon scattering coefficient $(g)$ of $1.8 \times 10^{-3}$ photon s $^{-1}$ atom $^{-1}$. The $g$-value was calculated 
Table 2

Magnesium to Sodium and Magnesium to Potassium Ratios in the Solar System

\begin{tabular}{lcl}
\hline \hline Object & $\mathrm{Mg} / \mathrm{Na}$ & \multicolumn{1}{c}{ Reference } \\
\hline Offset 8.8 $R_{E}$ & $<10$ & This work, Brown (2001) \\
Offset 14.4 $R_{E}$ & $<7$ & This work, Brown (2001) \\
Lunar exosphere & $<170$ & Stern et al. (1997), Stern (1999), Potter \& Morgan (1998) \\
Mercury exosphere & $0.05-2.5$ & Vervack et al. (2010) \\
Cosmic abundance & 18 & Cox \& Allen (1999) \\
CI chondrite & 19 & Lodders \& Fegley (1998) \\
Terrestrial oceans & 0.11 & Haynes (2012) \\
Terrestrial crust & 0.93 & Haynes (2012) \\
\hline Object & $\mathrm{Mg} / \mathrm{K}$ & \\
\hline Offset 8.8 $R_{E}$ & $<280$ & This work, Brown (2001) \\
Offset 14.4 $R_{E}$ & $<224$ & This work, Brown (2001) \\
Lunar exosphere & $<350$ & Stern et al. (1997), Stern (1999), Potter \& Morgan (1988) \\
Mercury exosphere & $2-20$ & Vervack et al. (2010), Potter et al. (2002) \\
Cosmic abundance & 275 & Cox \& Allen (1999) \\
CI chondrite & 176 & Lodders \& Fegley (1998) \\
Terrestrial oceans & 5.2 & Haynes (2012) \\
Terrestrial crust & 1.8 & Haynes (2012) \\
\hline
\end{tabular}

using the method described in Chamberlin \& Hunten (1987) using magnesium oscillator strengths from Haynes (2012) and solar flux from A'Hearn et al. (1983) Doppler shifted to account for Europa's heliocentric velocity at the time of the observations (see Table 1). These intensity limits imply upper limits for the magnesium column density of $2 \times 10^{10} \mathrm{~cm}^{-2}$ at $8.8 R_{E}$ and $9 \times 10^{9} \mathrm{~cm}^{-2}$ at $14.4 R_{E}$.

\section{CONCLUSIONS}

We searched archival HST-FOS spectra of Europa's atmosphere for the $2852 \AA$ magnesium emission feature. We were unable to detect magnesium and, instead, derive upper limits of $2 \times 10^{10} \mathrm{~cm}^{-2}$ at $8.8 R_{E}$ and $9 \times 10^{9} \mathrm{~cm}^{-2}$ at $14.4 R_{E}$ on the column abundance of magnesium. Comparison with the sodium and potassium column abundances measured by Brown (2001) yields upper limits to the magnesium to sodium ratio of $\sim 10$ at $8.8 R_{E}$ and $\sim 7$ at $14.4 R_{E}$ and upper limits to the magnesium to potassium ratio of $\sim 280$ at $8.8 R_{E}$ and $\sim 224$ at $14.4 R_{E}$ (see Table 2). The meteoritic (CI chondrites) and cosmic $\mathrm{Mg} / \mathrm{Na}$ are 19 and 18, respectively (Cox \& Allen 1999; Lodders \& Fegley 1998); thus it appears that Europa's atmospheric $\mathrm{Mg} / \mathrm{Na}$ is at least a factor of two lower than meteoritic and cosmic abundances. The upper limit on $\mathrm{Mg} / \mathrm{K}$ in Europa's atmosphere is also less than cosmic.

The lower than cosmic abundance of magnesium in Europa's atmosphere has two possible explanations, either magnesium is not present in surface concentrations sufficient to result in a detection in Europa's atmosphere or magnesium is abundant on the surface but is being sputtered into Europa's atmosphere at a lower rate than sodium and potassium. Recent models of spectra of Europa's surface generally include magnesium and sodium sulfate salts (such as hexahydrite $\left(\mathrm{MgSO}_{4} \cdot 6 \mathrm{H}_{2} \mathrm{O}\right)$, mirabilite $\left(\mathrm{Na}_{2} \mathrm{SO}_{4} \cdot 10 \mathrm{H}_{2} \mathrm{O}\right)$, and bloedite $\left.\left(\mathrm{Na}_{2} \mathrm{Mg}\left(\mathrm{SO}_{4}\right)_{2} \cdot 4 \mathrm{H}_{2} \mathrm{O}\right)\right)$ in $\mathrm{Mg} /$ $\mathrm{Na}$ ratios that are lower than our calculated upper limits for Europa's atmosphere (Dalton et al. 2005, 2012; Dalton 2007; Dalton \& Pitman 2012). Models of Europa's ocean result in high concentrations of both magnesium and sodium with a $\mathrm{Mg} / \mathrm{Na}$ ratio that is approximately half of our calculated upper limit (Zolotov \& Kargel 2009).

However, it is also possible that a significant amount of magnesium is present on Europa's surface but it is not being sputtered into the atmosphere. Although magnesium has recently been detected in the atmosphere of Mercury (McClintock et al. 2009), it has not been detected in the sputtered atmospheres of the Moon (Stern et al. 1997) and Io (Na et al. 1998). The $\mathrm{Mg} / \mathrm{Na}$ and $\mathrm{Mg} / \mathrm{K}$ ratios for the exospheres of Mercury and the Moon are shown in Table 2. Since the abundance of magnesium in the lunar regolith is known, it is possible to model the magnesium abundance in an atmosphere created by sputtering. Based on a stoichiometric atmosphere model (Flynn \& Stern 1996), Stern et al. (1997) find that the magnesium in the lunar atmosphere is depleted by at least factor of 10 based on the known lunar surface composition. The discrepancy between the known abundances of magnesium on the lunar surface and the inability to detect magnesium in the lunar atmosphere has yet to be explained. Although magnesium's presence in the atmosphere of Mercury is not surprising given that magnesium bearing minerals exist on the surface, the relative abundance and distribution of magnesium compared to sodium and calcium indicate that sputtering processes may be different for different minerals or atoms (McClintock et al. 2009; Vervack et al. 2010). The lunar and Mercurian examples suggest that magnesium may sputter less efficiently than sodium and potassium, a property that has been demonstrated experimentally for some Europa relevant salts; sodium and potassium sulfates are more easily sputtered than magnesium sulfates (McCord et al. 2001). This difference in sputtering efficiency could result in an enhancement of magnesium salts on the surface relative to potassium and sodium salts and a corresponding depletion in magnesium relative to potassium and sodium in Europa's atmosphere. Although the sodium in Europa's atmosphere is mostly endogenic (Brown 2001), it is possible that Iogenic sodium implanted on Europa's surface could also affect the ratio.

The upper limit we have calculated on the $\mathrm{Mg} / \mathrm{Na}$ ratio in Europa's atmosphere is lower than ratio in CI chondrites by at least a factor of two, which suggests that the trace elements observed in Europa's atmosphere are not meteoritic in origin, assuming similar sputtering efficiencies. However, for the upper limit on the magnesium abundance to provide a stronger constraint on the surface composition, and its origin, more stringent upper limits from future observations are required.

This work was initially supported by a Summer Undergraduate Research Fellowship from the California Institute of 
Technology funded by a trust established by J. Edward Richter. S.M.H. is supported by NSF Astronomy and Astrophysics Postdoctoral Fellowship AST-1102827.

\section{REFERENCES}

A'Hearn, M. F., Ohlmacher, J. T., \& Schleicher, D. G. 1983, NASA STI/Recon Technical Report N, 84, 20465

Anderson, J. D., Schubert, G., Jacobson, R. A., et al. 1998, Sci, 281, 2019

Brown, M. E. 2001, Icar, 151, 190

Brown, M. E., \& Hill, R. E. 1996, Natur, 380, 229

Carlson, R. W., Anderson, M. S., Johnson, R. E., Schulman, M. B., \& Yavrouian, A. H. 2002, Icar, 157, 456

Carlson, R. W., Calvin, W. M., Dalton, J. B., et al. 2009, in Europa, ed. R. T. Pappalardo, W. B. McKinnon, \& K. K. Khurana (Tucson, AZ: Univ. Arizona Press), 283

Carlson, R. W., Johnson, R. R., \& Anderson, M. S. 1999, Sci, 286, 97

Carlson, R. W., Weissman, P. R., Smythe, W. D., \& Mahoney, J. C. 1992, SSRv, 60,457

Carr, M. H., Belton, M. J. S., Chapman, C. R., et al. 1998, Natur, 391, 363

Chamberlin, J. W., \& Hunten, D. M. 1987, Theory of Planetary Atmospheres (San Diego: Academic Press)

Cox, A. N., \& Allen, C. N. 1999, Astrophysical Quantities (New York: Springer) Dalton, J. B. 2007, GeoRL, 34, L21205

Dalton, J. B., Prieto-Ballesteros, O., Kargel, J. S., et al. 2005, Icar, 177, 472

Dalton, J. B., III, \& Pitman, K. M. 2012, JGRE, 117, 9001

Dalton, J. B., III, Shirley, J. H., \& Kamp, L. W. 2012, JGRE, 117, 3003
Flynn, B. C., \& Stern, S. A. 1996, Icar, 124, 530

Haynes, W. C. 2012, CRC Handbook of Chemistry and Physics (Boca Raton: CRC Press)

Kivelson, M. G., Khurana, K. K., Russell, C. T., et al. 2000, Sci, 289, 1340

Lane, A. L., Nelson, R. M., \& Matson, D. L. 1981, Natur, 292, 38

Lodders, K., \& Fegley, B. 1998, The Planetary Scientist's Companion (Oxford: Oxford Univ. Press)

McClintock, W. E., Vervack, R. J., Bradley, E. T., et al. 2009, Sci, 324, 610

McCord, T. B., Hansen, G. B., Fanale, F. P., et al. 1998, Sci, 280, 1242

McCord, T. B., Orlando, T. M., Teeter, G., et al. 2001, JGR, 106, 3311

McGrath, M. A., Hansen, C. J., \& Hendrix, A. R. 2009, in Europa, ed. R. T. Pappalardo, W. B. McKinnon, \& K. K. Khurana (Tucson, AZ: Univ. Arizona Press), 485

Na, C. Y., Trafton, L. M., Barker, E. S., \& Stern, S. A. 1998, Icar, 131, 449

Paranicas, C., Carlson, R. W., \& Johnson, R. E. 2001, GeoRL, 28, 673

Paranicas, C., Ratliff, J. M., Mauk, B. H., Cohen, C., \& Johnson, R. E. 2002, GeoRL, 29, 18

Potter, A. E., Anderson, C. M., Killen, R. M., \& Morgan, T. H. 2002, JGRE, 107, 5040

Potter, A. E., \& Morgan, T. H. 1988, Sci, 241, 675

Potter, A. E., \& Morgan, T. H. 1998, JGR, 103, 8581

Stern, S. A. 1999, RvGeo, 37, 453

Stern, S. A., Parker, J. W., Morgan, T. H., et al. 1997, Icar, 127, 523

Strazzulla, G., Baratta, G. A., Leto, G., \& Gomis, O. 2007, Icar, 192, 623

Vervack, R. J., McClintock, W. E., Killen, R. M., et al. 2010, Sci, 329,672

Zolotov, M. Y., \& Kargel, J. S. 2009, in Europa, ed. R. T. Pappalardo, W. B. McKinnon, \& K. K. Khurana (Tucson, AZ: Univ. Arizona Press), 431 